\title{
Multiple cerebral gliomas in multiple sclerosis
}

\author{
T. J. REAGAN AND I. S. FREIMAN \\ From the Mayo Clinic and Mayo Foundation, Rochester, Minnesota, \\ and the Goldwater Memorial Hospital, New York, N.Y., U.S.A.
}

SUMMARY A patient with multiple sclerosis and a multicentric anaplastic astrocytoma is described. The evidence suggests that the relationship is not coincidental and that the astrocytomas originate in multiple sclerosis plaques.

When two disease processes, each of unknown cause, occur in the same patient, exploration of their possible relationship is warranted. The concurrence of cerebral glioma and multiple sclerosis has been documented in seven previously reported cases (Scherer, 1938; MunchPetersen, 1949; Zimmerman and Netsky, 1950; Brihaye, Périer, and Sténuit, 1963; Russell and Rubinstein, 1971) and suggested in one other (Herndon, Rubenstein, Freeman, and Mathieson, 1970). In the following report, the eighth case of these two conditions occurring in a patient is recorded.

\section{CASE REPORT}

In 1965 , a 40 year old woman had had difficulty in using her right hand. In 1967, weakness gradually developed, initially of her left leg and later of her right leg. The weakness increased to the degree that she fell at times and needed a cane to walk. In 1968, she was hospitalized elsewhere for evaluation. Multiple sclerosis, apparently, was diagnosed, and she was discharged to the home care service during which time the weakness increased until she became confined to a wheelchair. In July 1970 she developed weakness of her right arm, aphasia, and confusion, as well as faecal and urinary incontinence. She was then readmitted to hospital.

Examination at that time revealed an anxious woman who had difficulty in following commands. There was a right homonymous hemianopia with bilateral temporal optic disc pallor, horizontal nystagmus on right lateral gaze, rotary nystagmus on left lateral gaze, and a mild paresis of the right lower portion of her face. The cranial nerves were otherwise intact. Mild to moderate weakness was noted in both upper extremities, especially the right.
The legs were paralysed. Muscle tone was increased in all limbs. Response to pinprick was decreased in both legs, especially distally. The tendon reflexes were active bilaterally, with flexion withdrawal of both lower limbs on plantar stimulation. There was no Babinski response. Lumbar puncture yielded clear cerebrospinal fluid with no cells. The protein level was $180 \mathrm{mg} / 100 \mathrm{ml}$., and the VDRL was nonreactive. The patient was treated with prednisone for two weeks, but did not improve. Other pertinent past history included diabetes mellitus of more than 20 years' duration in which treatment involved insulin and tolbutamide. She had had syphilis in 1940 , and presumably had been treated; the VDRL test had been positive at 1:2 dilution. She had a history of labile hypertension of unknown duration. She was then transferred to a second hospital for further care.

Physical examination on admission disclosed a chronically ill, dull woman. Her pulse rate was $100 /$ min and regular; blood pressure $130 / 90 \mathrm{~mm} \mathrm{Hg}$ and temperature $37 \cdot 8^{\circ} \mathrm{C}\left(100^{\circ} \mathrm{F}\right)$. She was sluggish in answering questions. Pupils were equal and reactive to light and near vision. No papilloedema was present.

Shortly after admission, her temperature increased to between 38.3 and $38.9^{\circ} \mathrm{C}\left(101-102^{\circ} \mathrm{F}\right)$. A urine culture grew $\alpha$-streptococci and Proteus organisms, and she was given ampicillin. She continued to be febrile and gradually became drowsy and lethargic. On the seventh day after admission, mild ketoacidosis developed. Insulin and fluids were administered, and gentamicin was given. The ketoacidosis improved, but the patient remained febrile. On the 11 th day of hospitalization, the patient was obtundent. On the 16th day of hospitalization, there was questionable neck rigidity; a spinal tap revealed clear fluid with an opening pressure of $23 \mathrm{~cm}$ of water; there were no cells, the protein level was $130 \mathrm{mg} /$ 


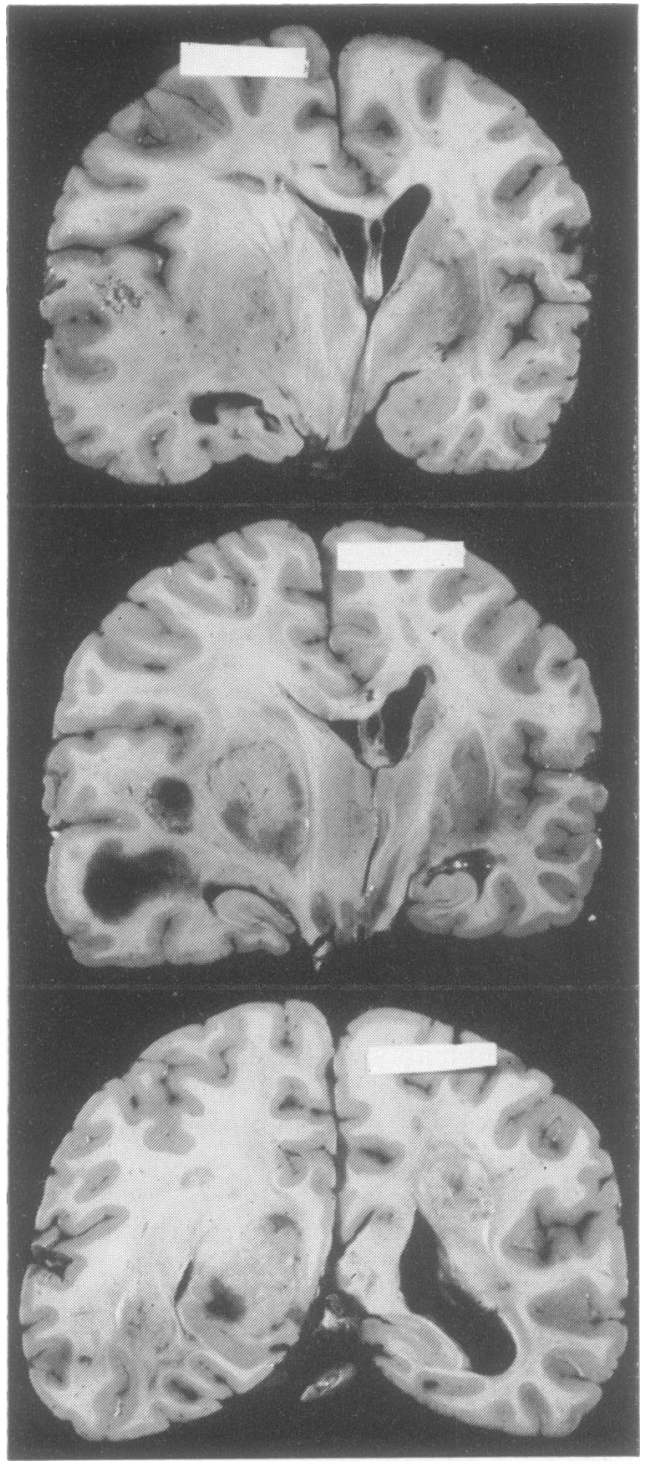

FIG. 1. Coronal sections through cerebral hemispheres showing tumours and plaques at levels of anterior thalamus (top); midthalamus (middle); and pulvinar (bottom).

$100 \mathrm{ml}$., and the sugar level was $167 \mathrm{mg} / 100 \mathrm{ml}$. On the 18th day of hospitalization, the patient was comatose, but she reacted to painful and neckstretching stimuli. Occasional spontaneous movements of the left upper limb were observed. Pupils were in mid-dilatation and were not reactive to light. The right upper and lower extremities were flaccid.
The deep tendon reflexes were depressed on the right. Plantar stimulation resulted in contraction of the hamstring muscle bilaterally. The patient was treated with infusion of corticotropin (60 units every eight hours for eight days). During this time, she

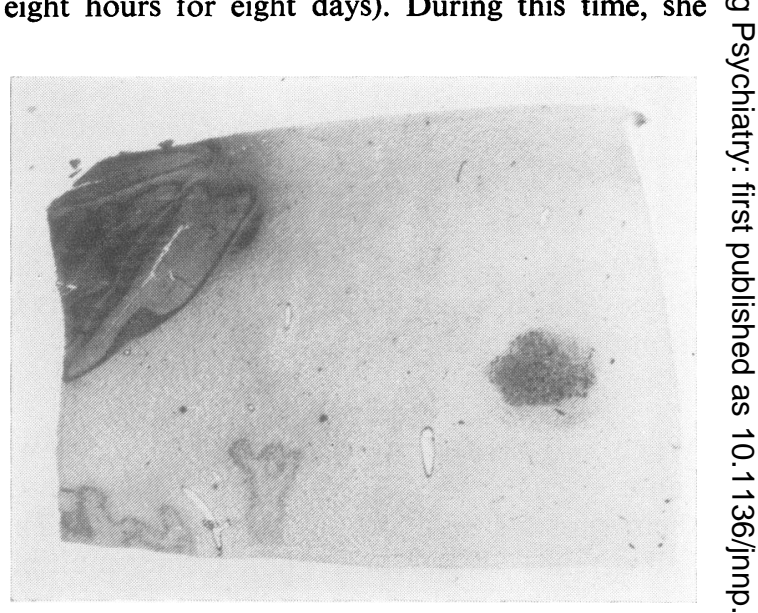

FIG. 2. Right cerebellar hemisphere section illus trating $5 \mathrm{~mm}$ tumour nodule in white matter (Holzer on reduced from $\times 3$.

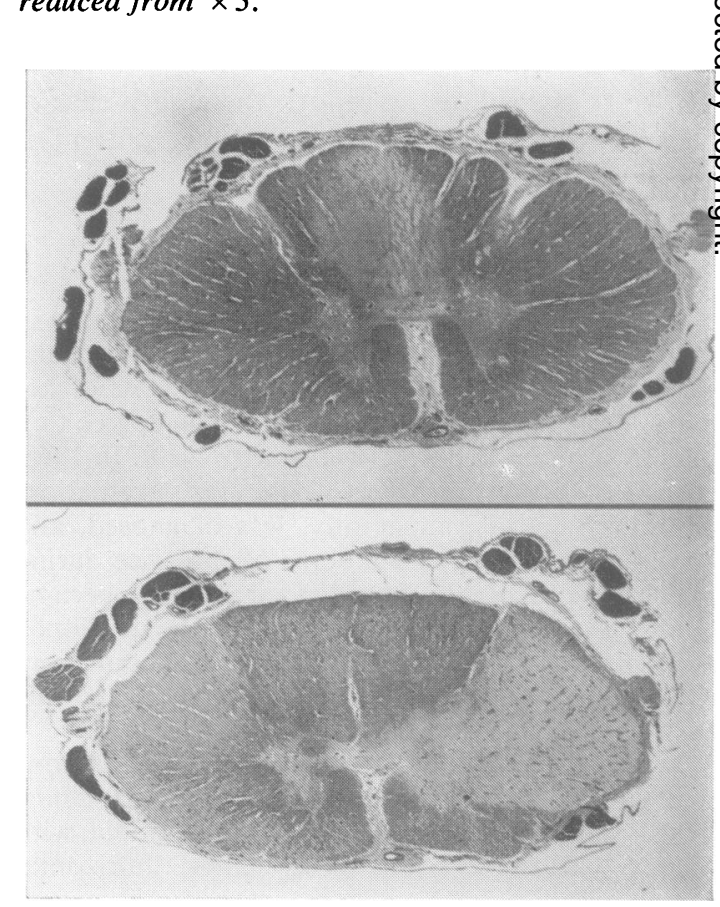

FIG. 3. Thoracic spinal cord sections showing multiple sclerosis plaques in posterior columns (top); and right lateral column (bottom). Luxol fast blue$P A S, \times 6$. 


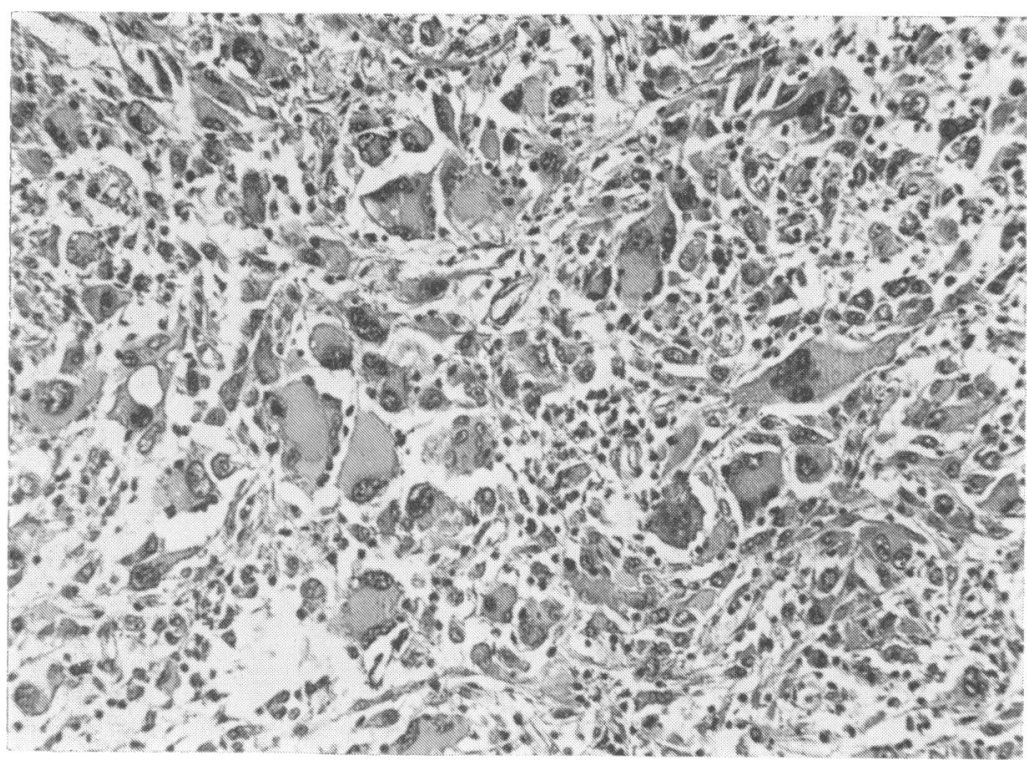

FIG. 4. Tumour section

from left ventricular nucleus. Haematoxylin and eosin, $\times 150$.

became afebrile and was more alert and attentive. On completion of the infusion, she again became febrile in spite of antibiotics. She gradually became more lethargic and finally comatose. She died 35 days after admission.

At necropsy, significant pathological findings outside the central nervous system were limited to pulmonary congestion with patchy bronchopneumonia. The brain weighed 1,450 g. External examination revealed extensive swelling of the posterior half of the left cerebral hemisphere and deep grooving of the left hippocampal gyrus, indicating tentorial herniation. The cerebral hemispheres were sectioned coronally at $1 \mathrm{~cm}$ intervals and the brain-stem and cerebellum were sectioned at $5 \mathrm{~mm}$ intervals.

Tumour masses, which were separate and somewhat circumscribed, were evident in both cerebral hemispheres, the corpus callosum, the brain-stem, and the cerebellum. The cerebral tumours are illustrated in Fig. 1. The largest mass occupied the left lenticular nucleus. Separated from this central mass by somewhat necrotic-appearing white matter were masses in the left parieto-occipital area, left temporal lobe, and the lower part of the left internal capsule. The mass in the left internal capsule extended through the left cerebral peduncle into the upper pons. A separate circumscribed tumour was present in the right parietal white matter, and two small tumour nodules were present in the corpus callosum. One nodule, $5 \mathrm{~mm}$ in diameter, was present in the right cerebellar hemisphere (Fig. 2). No tumour was seen in the spinal cord sectioned at 5 to $10 \mathrm{~mm}$ intervals.
Thus, gross inspection gave the impression of at least seven separate tumour masses in the central nervous system. The cut surface of the larger tumours showed irregular areas of necrosis and haemorrhage.

A second type of lesion also was seen distributed through the cerebrum (Fig. 1), brain-stem, and spinal cord (Fig. 3). These lesions were sharply bordered, variably shaped, grey translucent patches in the white matter, and ranged from a few millimetres to $2 \mathrm{~cm}$ in maximal diameter. These plaques outnumbered the tumour nodules, and many of them were removed from areas of tumour involvement. However, every tumour nodule except one (the cerebellar nodule) was grossly contiguous with at least one plaque.

Numerous sections from the involved areas of the nervous system were studied microscopically, and appropriate stains were employed to bring out special features. The grossly circumscribed tumours were pleomorphic in their cellular composition (Fig. 4). They contained a population of large cells, as large as $200 \mu$ in diameter, and a mixed population of smaller cells. The large cells, which dominated the histological picture, were irregular and contained abundant eosinophilic cytoplasm, often with elongated cytoplasmic processes. The nuclei were large, irregular, and often convoluted. Multiple nuclei were frequent. Eosinophilic inclusions of different sizes were seen in many of the nuclei. The inclusion material had the tinctorial characteristics of the cytoplasm. These inclusions probably represent 

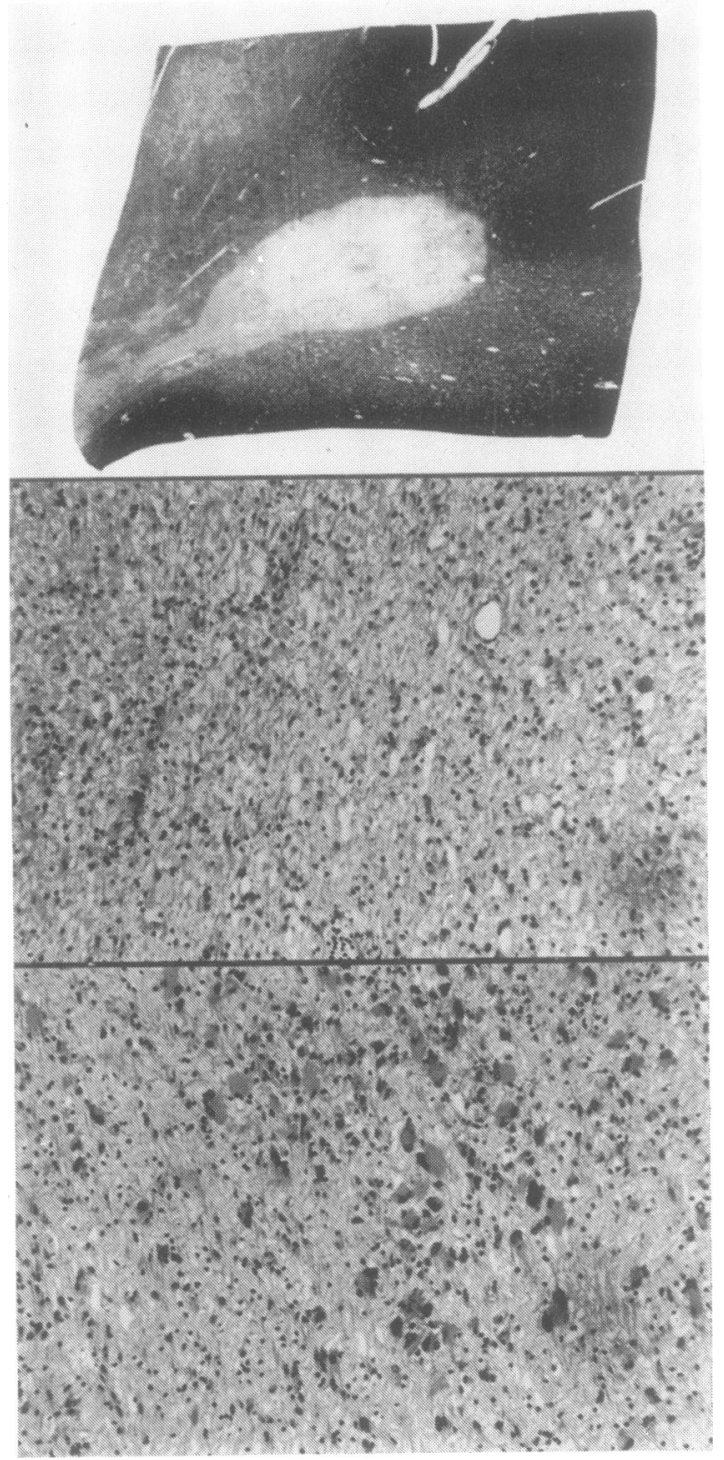

FIG. 5. Top: plaque from left frontal lobe. Luxol fast blue-cresyl violet, reduced from $\times 3$. Middle: regular astrocytic gliosis in top portion of plaque. Haematoxylin and eosin, reduced from $\times 150$. Bottom: collection of bizarre astrocytes in bottom portion of plaque. Haematoxylin and eosin, $\times 100$.

cytoplasmic invaginations cut in various planes that are often seen in tumour cells of this type (Robertson and MacLean, 1965).

The smaller cells were of various types. Most of them were astrocytes, somewhat resembling the giant cells, and demonstrating fibres stained with the phosphotungstic acid-haematoxylin and Holzer methods. The remaining small cells were lymphocytes, plasma cells, and microglia.

The tissue that appeared to be degenerating white matter, located between the circumscribed nodules in the left cerebral hemisphere, was infiltrated with more spindle-shaped neoplastic astrocytes. The tissue around the nodules in the corpus callosum and right hemisphere was also infiltrated with tumour cells, but connections between them could not be established histologically. The cerebellar nodule was clearly isolated.

The second type of lesion in the cerebrum, brainstem, and pons was a typical plaque of multiple sclerosis with a sharply circumscribed border, scarcity of myelin, good preservation of axis cylinders, and variable astrocytic gliosis.

Each tumour except the cerebellar nodule infiltrated one or more plaques, and the border between astrocytosis and astrocytoma was never clearly defined within the invaded plaques.

Two lesions warrant special mention. One of

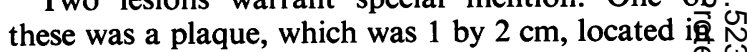
the left frontal white matter (Fig. 5, top). Abou $w$ three-fourths of the area of the plaque had the usual histological features of multiple sclerosis (Figs 5 , middle), with a slightly more intense perivascular lymphocytic infiltrate than other plaques in this case At one side of this plaque, however, the astrocyte were more numerous and larger, and many assume $\overrightarrow{0}$ the bizarre cytoplasmic and nuclear configuration of $\omega$ the giant cells of the previously described tumours (Fig. 5, bottom). The second particularly interesting lesion was in the optic chiasm (Fig. 6). The dorsal one-third of the chiasm showed a typical plaque of multiple sclerosis. The ventral one-third was $\stackrel{\circ}{\mathbb{Q}}$ occupied by tumour. These lesions were continuous $\stackrel{2}{\Rightarrow}$ on the left side of the chiasm. The left optic tract also was infiltrated by tumour.

\section{COMMENT}

The tumours in our patient were astrocytomas with extensive anaplastic change (glioblastoma multiforme). More descriptive terms such as 'giant cell glioblastoma' or 'glioblastoma gigantocellulare ganglioides' have been applied to this type of tumour. The gross appearance of multiplicity in gliomas may be deceptive and can be confirmed only by careful histological study of the intervening tissue (Batzdorf and $\mathrm{N}$ Malamud, 1963).

Although gross inspection revealed that many 
TABLE 1

REPORTED CASES OF MULTIPLE SCLEROSIS (MS) WITH CEREBRAL GLIOMA

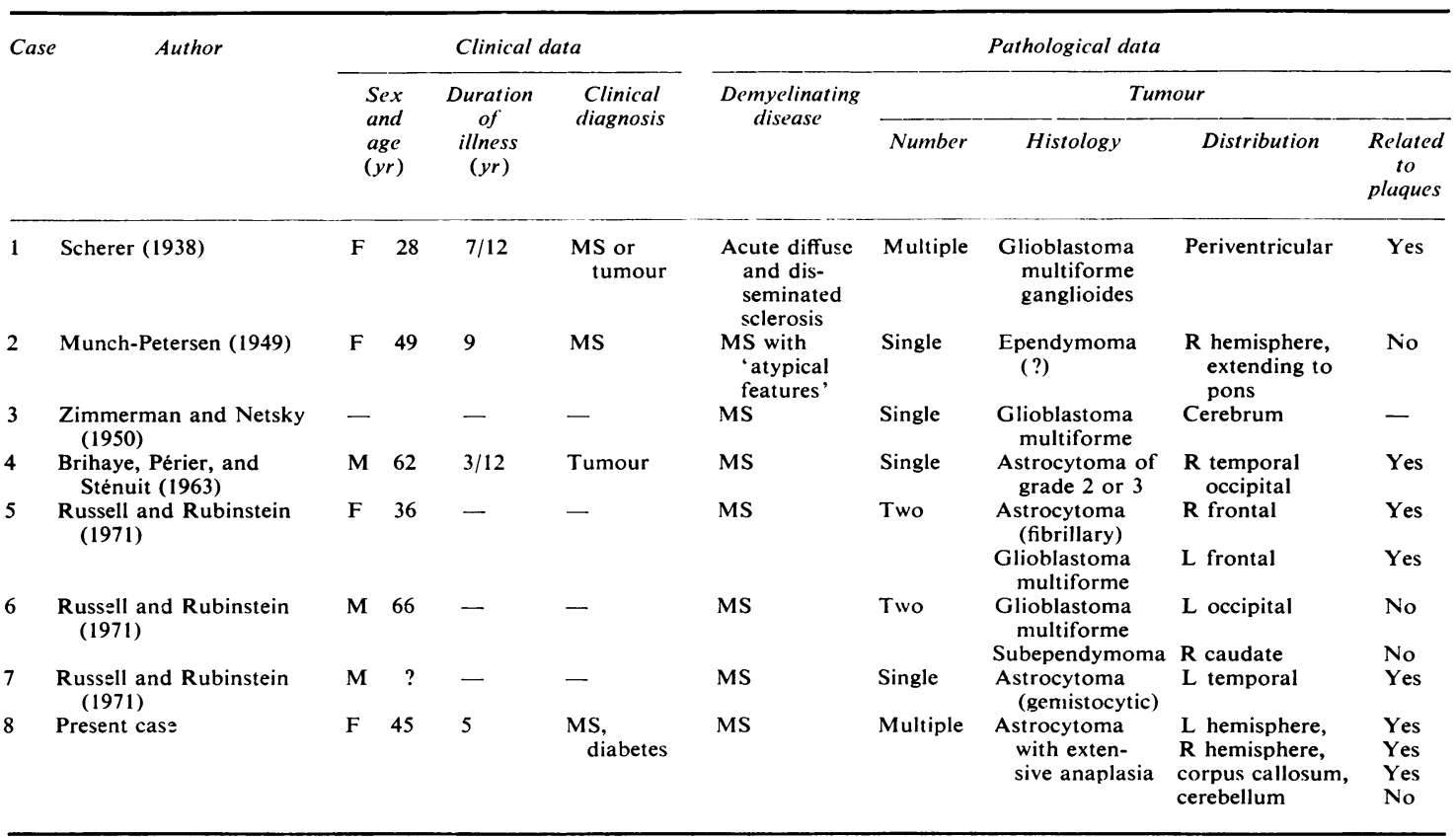

separate tumour masses existed in our patient, the four in the left hemisphere were connected by bridges of less anaplastic astrocytic neoplasm. A moderate infiltration of the corpus callosum with atypical astrocytes suggests that the two nodules located there may also be connected with the left hemispheric mass. No connection with the right hemisphere or the cerebellar tumours could be demonstrated. Thus, there were only three distinct gliomas, excluding the atypical plaque described in the left frontal lobe.

Cerebral glioma in association with multiple sclerosis has been reported in seven previous cases (Table 1). Many of the case descriptions
FIG. 6. Optic chiasm section showing plaque in dorsal portion and tumour in ventral and left lateral portions. Luxol fast bluecresyl violet, $\times 8$. 
are lacking in clinical or pathological detail. A case reported by Herndon et al. (1970) is not included because of some doubt, expressed by the authors, that the lesion they were describing in association with multiple sclerosis was, in fact, a neoplasm.

There are four possible ways in which two coexistent pathological conditions may be related. These hypothetical relationships, with specific reference to glioma and multiple sclerosis, are outlined in Table 2. The rarity of the concurrence of these two relatively common conditions would, at first glance, suggest that the relationship is merely coincidental. However, closer analysis of some of the pathological features outlined in Table 1 suggests a more intimate association.

TABLE 2

THEORETICAL RELATIONSHIPS OF MULTIPLE SCLEROSIS (MS) TO GLIOMA

\begin{tabular}{lll}
\hline Cause & Effect & Hypothetical mechanism \\
\hline Factor(s) A $\longrightarrow$ MS & Coincidence \\
Factor(s) B $\longrightarrow$ Glioma & $\begin{array}{l}\text { Bipotential agent (cytolytic-onco- } \\
\text { genic) such as Papova virus }\end{array}$ \\
Factor(s) C $\longrightarrow$ MS & Glioma & $\begin{array}{l}\text { Induction of allergy to CNS tissue } \\
\text { Neoplastic transformation of } \\
\text { reactive astrocytes }\end{array}$ \\
GS $\longrightarrow$ Glioma & \\
\hline
\end{tabular}

First, although the tumours have been described under various terms, all but one term may be considered to belong to the astrocytic series. The single exception, described by Munch-Petersen (1949) as a probable ependymoma, was considered by Russell and Rubinstein (1971), on the basis of the published illustrations, to be a glioblastoma. Thus, all the tumours in the series may be considered to be astrocytic. Although the number of cases is very small, the deviation from the usual distribution of cerebral gliomas among the various cytological types may be noted.

Second, and even more striking, is the observation that in four of the eight cases the gliomas were multiple. This incidence of $50 \%$ contrasts sharply with the incidence of $2.5 \%$ of multiple tumours in an unselected series of gliomas (Batzdorf and Malamud, 1963).
A third pertinent observation concerns the topographical relationship of tumour to the plaques. In the seven cases in which this information is available, a contiguous relationship between tumour and plaque was noted in five.

These observations may be subject to various interpretations but seem to favour the hypothesis that in a small percentage of patients with multiple sclerosis an unknown factor, hereditary or acquired, incites the reactive astrocytes of some plaques to undergo neoplastic transformation. It is difficult, if not impossible, from morphological observation, to determine whether a neoplasm is originating in a plaque or invading a plaque. The plaque shown in Fig. 5 could be interpreted as an illustration of the former. In either situation, it is not difficult to imagine that a multiple sclerosis plaque has been completely overgrown and obscured by a glioma. This may account for the failure to demonstrate a close topographical relationship between the two processes in some instances.

The authors wish to express their appreciation $t \stackrel{\overbrace{}}{\mathbb{Q}}$ Dr. Adolph Berger, Chief of Medicine, Goldwat Memorial Hospital, for making the clinical data on this patient available for study.

\section{REFERENCES}

Batzdorf, U., and Malamud, N. (1963). The problem of multicentric gliomas. Journal of Neurosurgery, 20, 122-136. Brihaye, J., Périer, O., and Sténuit, J. (1963). Multiple sclerosis associated with a cerebral glioma. Journal of Neuropathology and Experimental Neurology, 22, 128-137.

Herndon, R. M., Rubinstein, L. J., Freeman, J. M., and $\overrightarrow{\vec{B}}$ Mathieson, G. (1970). Light and electron microscopic 윽 observations on Rosenthal fibres in Alexander's disease and in multiple sclerosis. Journal of Neuropathology and Experimental Neurology, 29, 524-551.

Munch-Petersen, C. J. (1949). A case of disseminated sclerosis and glioma of the brain in the same patient. Acta Psychiatrica et Neurologica, 24, 599-605.

Robertson, D. M., and MacLean, J. D. (1965). Nuclear inclusions in malignant gliomas. Archives of Neurology, 3 13, 287-296.

Russell, D. S., and Rubinstein, L. J. (1971). Pathology of Tumours of the Nervous System, p. 179. 3rd edn. Arnold: London.

Scherer, H. J. (1938). La 'glioblastomatose en plaques': sur les limites anatomiques de la gliomatose et des processus $\frac{D}{0}$ sclérotiques progressifs (sclérose en plaques, sclérose diffuse de Schilder, sclérose concentrique). Journal Belge $\mathrm{N}$ de Neurologie et de Psychiatrie, 38, 1-17.

Zimmerman, H. M., and Netsky, M. G. (1950). The path- N ology of multiple sclerosis. Association for Research in $\mathrm{N}$ Nervous and Mental Disease, Proceedings, 28, 271-312. 